\title{
The Use of an Innovative Film-Forming Wound Dressing in the Treatment of Radiation Dermatitis during Concomitant Chemotherapy
}

\author{
Iratxe Arranz Villandiego*, Sara Gonzalez Aramberri \\ Clinica IMQ Zorrozaurre, Bilbao, Spain \\ Email: *iratxearranzvillandiego@hotmail.com
}

How to cite this paper: Villandiego, I.A. and Aramberri, S.G. (2018) The Use of an Innovative Film-Forming Wound Dressing in the Treatment of Radiation Dermatitis during Concomitant Chemotherapy. Journal of Cancer Therapy, 9, 1048-1056. https://doi.org/10.4236/jct.2018.912086

Received: November 29, 2018 Accepted: December 26, 2018 Published: December 29, 2018

Copyright ( 92018 by authors and Scientific Research Publishing Inc. This work is licensed under the Creative Commons Attribution International License (CC BY 4.0).

http://creativecommons.org/licenses/by/4.0/

\begin{abstract}
There is currently no consensus regarding the management of acute cutaneous reactions emerging from a concurrent treatment of radio-and chemotherapy, the goal of this case study series is to provide scientific evidence based on clinical practice regarding the efficacy of a film-forming wound dressing for patients receiving combined cancer therapy, in particular examples where the reactions are expected to be intense. In all the described cases, management of acute skin reactions allowed the cancer therapy to be finalized, instead of being interrupted due to an increase in the severity of the reactions. It has been shown in the current literature that the lack of compliance with treatment plans correlates with poorer clinical outcome. Evidence from the cases presented suggest that the studied dressing is safe and efficacious in the treatment and the prevention of acute cutaneous reactions arising from combined cancer therapy.
\end{abstract}

\section{Keywords}

Radiation Dermatitis, Film-Forming Wound Dressing, Skin Care

\section{Introduction}

Radiation dermatitis is one of the most common side effects of radiotherapy (RT) that affects up to $95 \%$ patients receiving treatment. Generally, radiation acute skin reaction scan arise shortly after the start of radiation therapy (from 2 to 4 weeks); this can vary depending on the magnitude of the administered radiation and its severity intensifies over time, directly associated to the accruing radiation on the tissues [1] [2].

There are several described risk factors that contribute to the exacerbation of 
skin reactions. Among these, concomitant treatment with chemotherapeutic agents is known to deteriorate skin conditions. Some medications have been well described to sensitize patients to radiation. Adjuvant chemotherapy is therefore accepted to substantially increase the risk to develop severe skin injuries [3] [4].

As there is currently no consensus regarding the management of acute cutaneous reactions emerging from a concurrent treatment of radio- and chemotherapy, the goal of this case study series is to provide scientific evidence based on clinical practice regarding the efficacy of an innovative film-forming wound dressing for patients receiving combined cancer therapy, in particular examples where the reactions are expected to be intense.

\section{Methods}

This study was designed as a case study series to explore the use of an innovative film-forming wound dressing (StrataXRT ${ }^{\oplus}$-Stratpharma AG, Switzerland) in an experimental setting using a variety of methods to obtain more in-depth knowledge about the use of this technology in the treatment of radiation dermatitis during concomitant chemotherapy. This medical device is already being used in the clinic (IMQ Zorrotzaurre in Bilbao, Spain) for treating patients that develop a significant erythema (degree II+), pruritos and/or desquamation and usually if they still had $20 \%-50 \%$ of the RT plan to be completed, but there is a lack of knowledge in its efficacy for patients receiving concomitant chemotherapy.

As the boundaries between the evolution of radiation dermatitis and context of RT and chemotherapy are not clearly evident and it was presumed that there would be more variables of interest than data points, this study was designed to reflect a qualitative investigation of different types of cancer locations. The data was captured using interviews, questionnaires and observations by the experienced oncology nursing team. All patients were enrolled and consented in the RT nursery consultation that is performed on a weekly basis to all patients receiving RT.

All participating patients were assessed on a weekly basis and up to 2 weeks after RT finalization by the same investigator to monitor improvement or record any adverse event.

\section{Results}

5 patients were prospectively recruited for this case study. No adverse events related to the studied product was noted throughout the investigation. Clinical and demographical features are summarized in Table 1.

Case 1: An innovative wound dressing for acutecutaneous reactions to concomitant radio- and chemotherapy for tongue base cancer

A 50-year old man diagnosed with epidermoid tongue base cancer with an extension to the tonsillar pillar was treated with a first line of adjuvant chemotherapy ( 3 cycles of Taxol-Cisplatin-5FU) followed by a second line of 
Table 1. Clinical and demographic characteristics of the study participants.

\begin{tabular}{|c|c|c|c|c|c|c|c|}
\hline \multirow[b]{2}{*}{$\begin{array}{l}\text { Patient } \\
\text { number }\end{array}$} & \multirow[b]{2}{*}{ Age } & \multirow[b]{2}{*}{ Sex } & \multirow[b]{2}{*}{ Cancer } & \multicolumn{3}{|c|}{ RT plan } & \multirow[b]{2}{*}{ Chemotherapy plan } \\
\hline & & & & $\begin{array}{c}\text { Total } \\
\text { Gy }\end{array}$ & Fractions & $\begin{array}{l}\text { Gy per } \\
\text { fraction }\end{array}$ & \\
\hline 1 & 50 & Male & $\begin{array}{l}\text { Tongue base } \\
\text { carcinoma with } \\
\text { extension to } \\
\text { the tonsillar pillar }\end{array}$ & 70 & 33 & 2.1 & $\begin{array}{l}\text { 3 cycles of } \\
\text { Taxol-Cisplatin-5FU) followed } \\
\text { by weekly Taxol-Carboplatin }\end{array}$ \\
\hline 2 & 69 & Female & $\begin{array}{l}\text { Rectum } \\
\text { neoplasia }\end{array}$ & 50.4 & 28 & 1.8 & $\begin{array}{l}6 \text { cycles of } 5 \mathrm{FU} \text { continuous } \\
\text { infusion for } 5 \text { days }\end{array}$ \\
\hline 3 & 53 & Female & $\begin{array}{l}\text { Submaxillary } \\
\text { gland neoplasia }\end{array}$ & 66 & 33 & 2 & $\begin{array}{l}2 \text { cycles of Cisplatin } \\
\text { every } 21 \text { days }\end{array}$ \\
\hline 4 & 58 & Male & $\begin{array}{l}\text { Tongue base } \\
\text { carcinoma }\end{array}$ & 70 & 35 & 2 & $\begin{array}{c}7 \text { cycles of } \\
\text { weekly Carboplatin }\end{array}$ \\
\hline 5 & 55 & Male & $\begin{array}{l}\text { Rectum } \\
\text { neoplasia }\end{array}$ & 54 & 30 & 1.8 & $\begin{array}{l}2 \text { cycles of Mitomycin and } \\
5 \mathrm{FU} \text { continuous infusion } \\
\text { for } 5 \text { out of every } 21 \text { days }\end{array}$ \\
\hline
\end{tabular}

chemotherapy (weekly Taxol-Carboplatin) and RT, consisting of VMAT radiation in the tongue base with a total dose of $70 \mathrm{~Gy}$ in 33 fractions, corresponding to $2.1 \mathrm{~Gy} /$ fraction.

After 18 sessions of RT the patient started showing signs of dry desquamation and an intense erythema in the neck area coupled with intense itching. Twicedaily treatment with the innovative film-forming wound dressing was started at this time point.

After 7 days from topical treatment start (23 RT sessions), nursing consultation was carried out to confirm a persistent erythema and a lower degree of pruritus (Figure 1(a)). No progression to more severe cutaneous toxicity was assessed.

14 days after treatment start with the wound dressing (27 RT sessions) erythema in the neck area did not worsen from the previous assessment, however suppuration areas on the left side of the neck and chin emerged (Figure 1(b)). Antibiotic treatment was therefore added.

21 days after wound dressing treatment start (31 RT sessions) a decreased erythema was present, with dry desquamation and signs of epidermal regeneration (Figure 1(c)). The patient also reported an improvement with regards to pruritus. It is critical to mention how overall cutaneous symptoms improved at this stage of radiotherapy, as we expect from our clinical experience that the signs of $\mathrm{RD}$ worsen with incremental doses. The film-forming wound dressing had been used in monotherapy and we consider it the reason for improvement.

The patient was then assessed 5 days after the end of RT ( 28 days from the start of topical treatment with the wound dressing). Dry desquamation in the neck area was considerably improved, although a section of the treated area presented a minor oozing. 


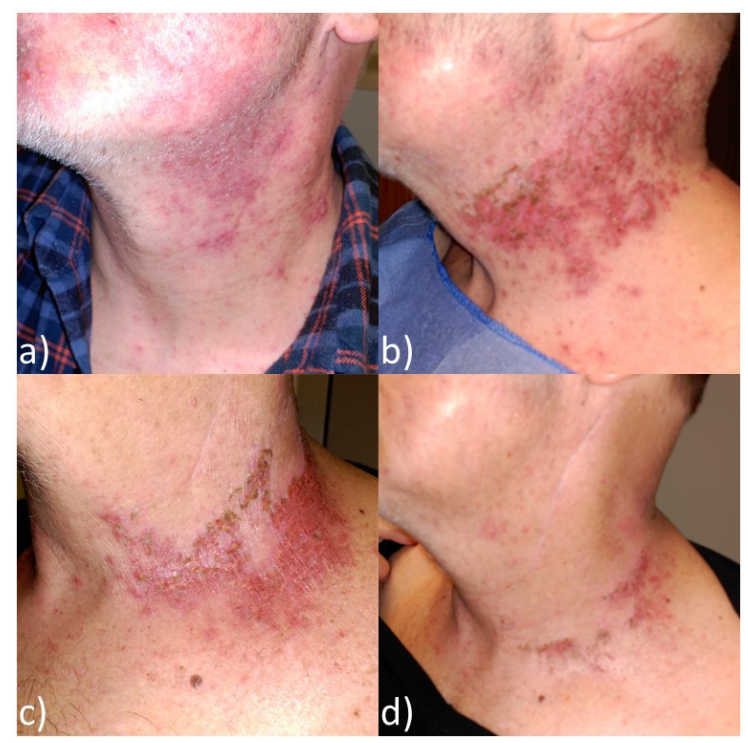

Figure 1. Radiation dermatitis evolution 7 days after wound dressing application and 23 RT sessions (a); 14 days after product application and 27 RT sessions (b); 21 days after product application and $31 \mathrm{RT}$ sessions (c); and 35 days after wound dressing application and 12 days after RT finalization (d).

12 days after RT finalization (35 days post topical treatment start) cutaneous lesions improved and reduced their extension (Figure 1(d)), therefore the innovative wound dressing was applied once daily. 7 days later the patient was discharged due to wound resolution.

Case 2: Treatment of acute dermatitis in patient undergoing concomitant radio- and chemotherapy for rectum cancer

A 69-year old woman was diagnosed with rectum neoplasia that was treated with RT and concomitant chemotherapy (6 cycles of 5FU continuous pump infusion for 5 days). The patient received VMAT radiation in the rectum area with a total dose of $50.4 \mathrm{~Gy}$ distributed in 28 fractions, corresponding to 1.8 Gy/fraction.

After 28 RT sessions, at treatment finalization, the patient underwent nurse consultation due to moist desquamation with minor exudate in the irradiated surface and intense pruritus in the perianal area (Figure 2(a)). Twice daily treatment with the innovative film-forming wound dressing was initiated.

8 days after treatment start with the wound dressing, an evaluation of the cutaneous reactions was carried out. The compromised area improved significantly and a reduction in pruritus was assessed (Figure 2(b)). Treatment with the film-forming wound dressing was reduced to once daily application.

15 days from the start of topical treatment the patient was discharged due to wound resolution (Figure 2(c)).

Case 3: Treatment of radiation dermatitis in patient undergoing concomitant radio- and chemotherapy for submaxillary gland cancer

A 53-year old woman diagnosed with submaxillary gland neoplasia was treated with surgery and chemotherapy ( 2 cycles of Cisplatin every 21 days) with 


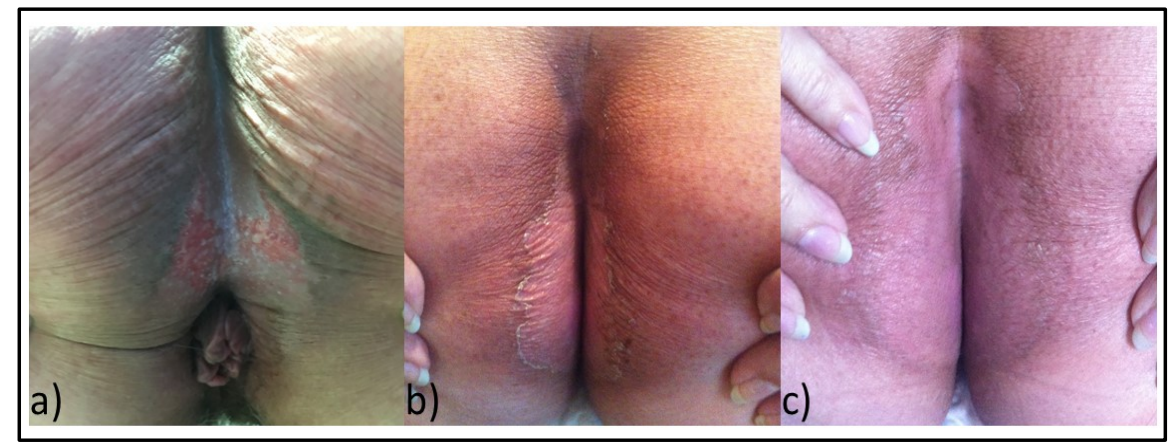

Figure 2. Radiation dermatitis at start with wound dressing application and end of RT (a); wound evolution at 8 days of product application (b) and 15 days of product application (c).

concomitant RT, consisting of VMAT with a total dose of 66 Gy divided in 33 sessions.

At RT session 17, twice-daily treatment with the innovative wound dressing was initiated due to intense erythema and pruritus in the irradiated area (Figure $3(\mathrm{a}))$.

7 days after topical treatment start (RT session 22), the patient still presented intense erythema and intense pruritus (Figure 3(b)). For this reason, twice daily Fluocinolone $0.01 \%$ cream was added to the topical therapy, 30 minutes prior to the wound dressing application.

14 days from the start of treatment with the film-forming wound dressing (RT session 26) the patient showed an improved erythema and a $3 \times 3 \mathrm{~cm}$ dry desquamation in the submandibular region (Figure 3(c)). For this reason, twice daily treatment with the wound dressing was continued, whereas Fluocinolone was suspended.

After 21 days from the start of topical treatment (corresponding to RT session 30) the patient was presenting a mild erythema with a minor dry desquamation in the irradiated area (Figure 3(d)). Treatment with the film-forming wound dressing was continued for 2 more days, after which the patient was followed up in an outpatient (dermatology) setting.

Case 4: Treatment of radiation dermatitis in patient undergoing concomitant radio- and chemotherapy for tongue base cancer

A 58-year old patient diagnosed with tongue base carcinoma, was treated with surgery and chemotherapy (7 cycles of weekly Carboplatin) and concomitant VMAT RT in the tongue base with a total dose of 70 Gy divided in 35 fractions.

After 21 RT sessions the patient underwent nurse consultation with an intense erythema and patchy moist desquamation on the right side of the neck (Figure 4(a)). Twice daily treatment with the innovative wound dressing was therefore initiated.

10 days later (RT session 31) the overall cutaneous conditions were substantially improved, presenting a minor erythema coupled to an extended desquamation (Figure 4(b)). The same topical treatment regimen was continued. 


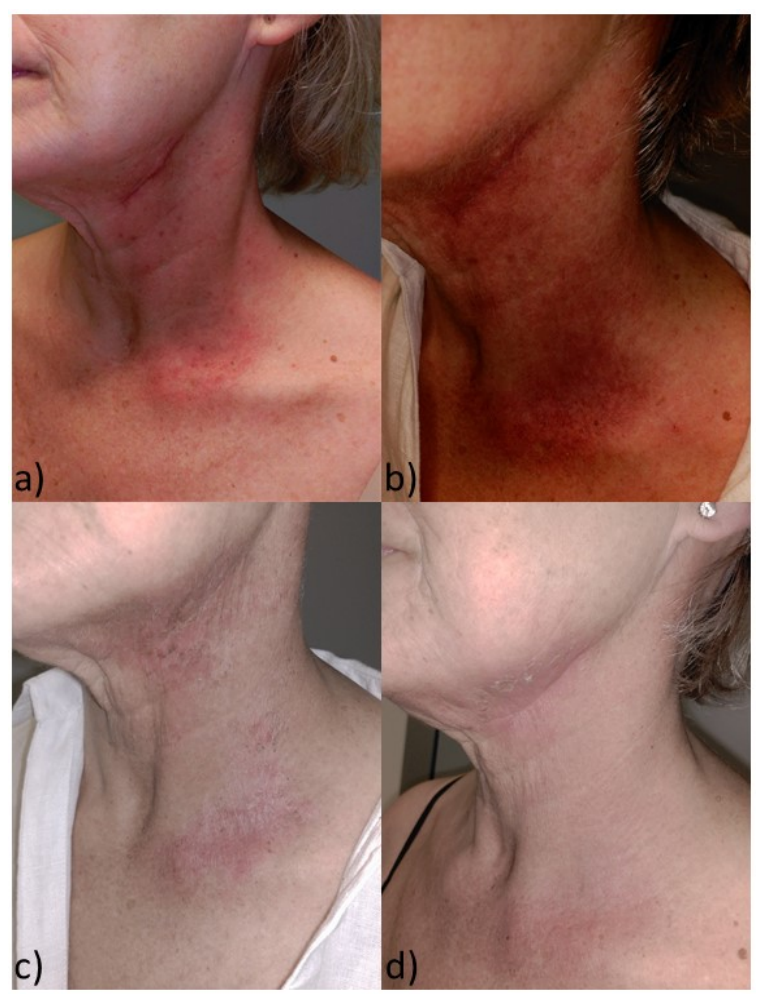

Figure 3. Radiation dermatitis at start with wound dressing application and after 17 RT sessions (a); wound evolution 7 days after product application and 22 RT sessions (b); 14 days after product application and $26 \mathrm{RT}$ sessions (c) and 21 days after product application and 30 RT sessions (d).

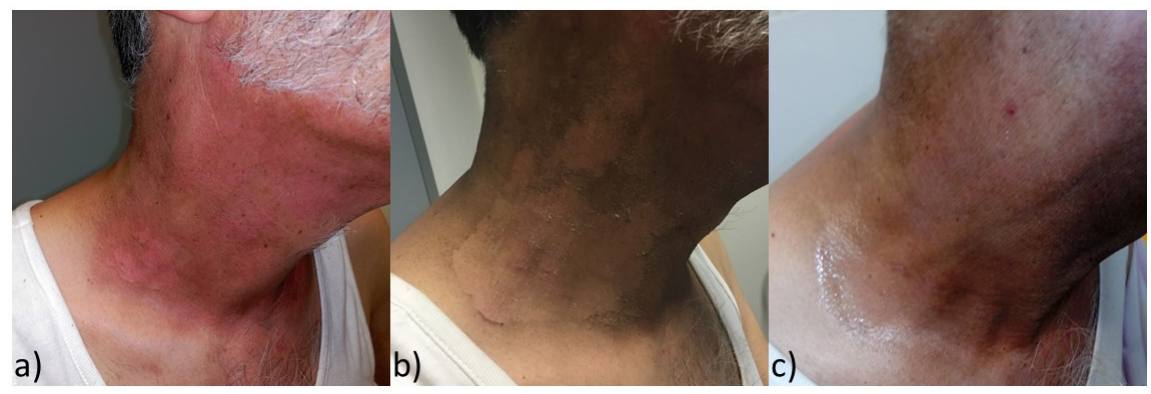

Figure 4. Radiation dermatitis at start with wound dressing application and after $21 \mathrm{RT}$ sessions (a); wound evolution 10 days after product application and 31 RT sessions (b); and 18 days after product application and 4 days after RT finalization (c).

18 days from treatment start with the film-forming wound dressing and 4 days post RT finalization the patient was discharged due to favorable wound evolution (Figure 4(c)). The current case was particularly striking, as the stand-alone use of the studied dressing enabled a reduction of the overall cutaneous toxicity during ongoing radiation therapy.

Case 5: Treatment of acute cutaneous reactions following concurrent radioand chemotherapy for anal cancer

A 55-year old man diagnosed with epidermoid carcinoma of the anal canal, was treated with chemotherapy ( 2 cycles of Mitomycin and 5FU in continuous 
pump infusion for 5 out of every 21 days) and concomitant RT, consisting of VMAT in the anal canal with a total dose of 54 Gy distributed in 30 fractions, corresponding to $1.8 \mathrm{~Gy} /$ fraction.

After 23 RT session the patient was assessed to have developed a moderate erythema and intense pruritus in the anal and pubic regions (Figure 5(a)). Topical treatment with the studied wound dressing was therefore started twice daily.

3 days later (RT session 24) amoderate erythema was still present frontally, as much as posteriorly (Figure 6(a)), and moist desquamation occurred in the groin and scrotum area. Topical treatment regimen was maintained without changes.

10 days after topical treatment start (RT session 26) a clear resolution of erythema occurred, however the patient presented moist desquamation in the groin and anal regions, and a dry desquamation on the buttocks (Figure 6(b)).

2 days later, a substantial overall improvement was observed; the extension of the inguinal moist desquamation was considerably reduced (Figure 5(b)). Dry desquamation in the anal region was instead unchanged.

7 days later (24 days from topical treatment start and 9 days from RT finalization) a control visit was carried out in order to assess the wound evolution; the patient was discharged due to wound resolution (Figure 5(c) and Figure 6(c)).

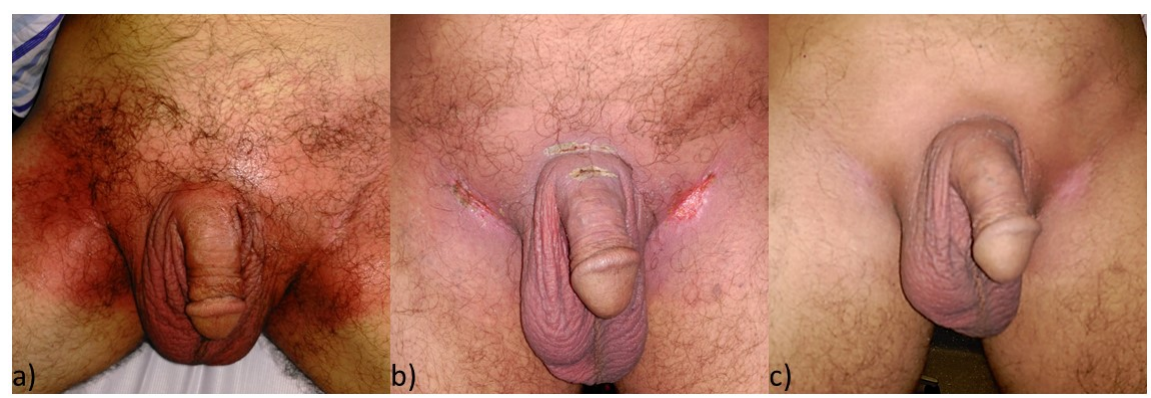

Figure 5. Evolution of cutaneous reactions in the pubic area at treatment start with the wound dressing and after 23 RT sessions (a); 18 days after product application and 2 days from RT finalization (b) and 24 days after product application and 9 days from RT finalization (c).

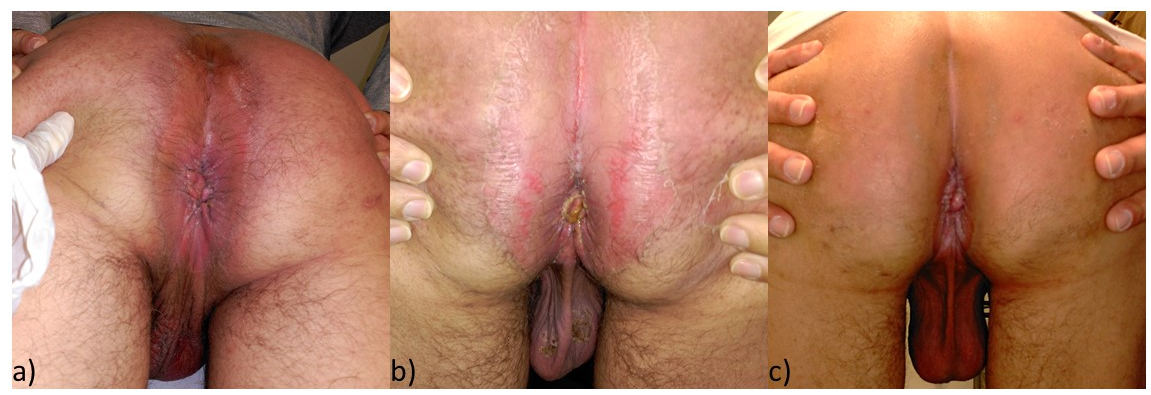

Figure 6. Evolution of cutaneous reactions in the perianal area after 3 days applying the wound dressing and after 24 RT sessions (a), 10 days after product application and 26 RT sessions (b) and 24 days of topical treatment and 9 days from RT finalization (c). 


\section{Discussion}

To the authors' knowledge, the use of the studied wound dressing in the form of a gel, as described in this case study series, shows first time evidence of reducing the severity of acute cutaneous reactions arising from ongoing radiation therapy with concomitant chemotherapy.

Evidence arising from daily clinical practice suggests that cutaneous reactions following radiation therapy can frequently affect oncological patients. Such reactions are particularly exacerbated with adjuvant chemotherapy and are commonly expected to deteriorate with the progression of the therapy. In all the described cases, management of acute skin reactions allowed the cancer therapy to be finalized, instead of being interrupted due to an increase in the severity of the reactions. It has been shown in the current literature that the lack of compliance with treatment plans correlates with poorer clinical outcomes [5]. The avoidance of cancer therapy interruptions enables an increased therapy efficacy, with direct benefits for the patient.

In all cases described above the studied wound dressing served as a self-leveling and film-forming dressing preventing trans-epidermal water loss and enabled a moist wound healing environment that is known to promote faster reepithelialisation and reduce the skin's acute inflammatory response [6].

A critical consideration needs to be pointed out from this case series: it is noteworthy how the wound dressing was used with similarly excellent efficacy and safety results across several types of cancer localizations. The flexible nature of the gel allows a perfect adaptation to all those body surfaces in which common physical sheeting are unsuccessful or impossible to use, such as those with high mobility (joints), high friction (axilla), wetsk in (mucosa), and higher hygienic necessities (perineum) [6].

Evidence from the cases presented above and from everyday clinical experience suggests that the studied dressing is safe and efficacious in the treatment and the prevention of acute cutaneous reactions arising from combined cancer therapy. Nevertheless, clinical trials on a larger sample size would be needed to confirm these preliminary findings.

\section{Conflicts of Interest}

The authors declare no conflicts of interest regarding the publication of this paper.

\section{References}

[1] Naylor, W. and Mallett, J. (2001) Management of Acute Radiotherapy Induced Skin Reactions: A Literature Review. European Journal of Oncology Nursing, 5, 221-233. https://doi.org/10.1054/ejon.2001.0145

[2] Porock, D. and Kristjanson, L. (1999) Skin Reactions during Radiotherapy for Breast Cancer: The Use and Impact of Topical Agents and Dressings. European Journal of Cancer Care, 8, 143-153.

https://doi.org/10.1046/j.1365-2354.1999.00153.x 
[3] Brown, K.R. and Rzucidlo, E. (2011) Acute and Chronic Radiation Injury. Journal of Vascular Surgery, 53, 15S. https://doi.org/10.1016/j.jvs.2010.06.175

[4] Hymes, S.R., Strom, E.A. and Fife, C. (2006) Radiation Dermatitis: Clinical Presentation, Pathophysiology, and Treatment. Journal of the American Academy of Dermatology, 54, 28-46. https://doi.org/10.1016/j.jaad.2005.08.054

[5] Ohri, N., Rapkin, B.D., Guha, C., Kalnicki, S. and Garg, M. (2016) Radiation Therapy Noncompliance and Clinical Outcomes in an Urban Academic Cancer Center. International Journal of Radiation Oncology, Biology, Physics, 95, 563-570. https://doi.org/10.1016/j.ijrobp.2016.01.043

[6] Quilis, A., Martín, J., Rodríguez, C., Sánchez, P. and Ribes, J.L. (2018) Reducing Radiation Dermatitis during Ongoing Radiation Therapy: An Innovative Film-Forming Wound Dressing. Journal of Radiation Oncology.

https://doi.org/10.1007/s13566-018-0356-5 